\title{
NT-proBNP correlates with the illness scores pneumonia severity index and CURB-65 in patients with pneumonia
}

\author{
Dario Martolini, Eleonora Pistella, Enrico Carmenini, Claudio Santini \\ Department of Internal Medicine, Vannini Hospital, Roma, Italy
}

\begin{abstract}
Pneumonia severity index (PSI) and CURB-65 (confusion, urea, respiratory rate, blood pressure, age $\geq 65$ ) are used to estimate the severity and prognosis of patients with pneumonia. NT-proBNP is a marker of myocardial stress and of sepsis-induced myocardial depression and might be used to predict short and long-term survival in patients with pneumonia. Twenty-three patients [age $79 \pm 15$ standard deviation (SD); M/F 8/15, CURB-65 2.2 \pm 0.9 SD, PSI $118 \pm 38$ SD, procalcitonin 3.9 \pm 5 SD] with pneumonia hospitalized in our Internal Medicine Unit were retrospectively evaluated. NT-proBNP was measured in the first 72 $\mathrm{h}$ of hospitalization. CURB-65 and PSI were calculated and correlation with biomarkers investigated. NT-proBNP showed a moderate statistically significant correlation with both PSI and CURB-65 (NT-proBNP $v s$ PSI, r $=0.42, \mathrm{P}<0.05, \mathrm{NT}-\mathrm{proBNP} v s$ CURB-65, $\mathrm{r}=0.46, \mathrm{P}<0.05$ ). These correlations were confirmed also when patients with a diagnosis of heart failure where excluded from the analysis, even if the correlation did not reach the statistical significance. NT-proBNP seems to well correlate with the illness scores PSI and CURB-65 and might be a reliable predictor of severity and survival in patients with pneumonia.
\end{abstract}

\section{Introduction}

Pneumonia is one of the most common infections all over the world, with a great burden on health system. UK statistics highlight every year between $0.5 \%$ and $1 \%$ of adults in the UK will have community-ac-

Correspondence: Dario Martolini, Department of Internal Medicine, Vannini Hospital, via Monte Grimano 73, 00138 Roma, Italy.

E-mail: martolini.dario@yahoo.it

Key words: Pneumonia; NT-proBNP; pneumonia severity index; CURB-65; illness score.

Contributions: DM and CS conceived the idea of the study. DM, EP and EC collected the data about every patient and helped to create a database. DM statistically analyzed the data and wrote down the first draft of the paper. EP, DM and CS revised the first draft and helped to produce the final version of the work.

Conflict of interest: the authors declare no potential conflict of interest.

Award: Premio Ricerca FADOI Lazio 2015 - $1^{\text {st }}$ place.

Received for publication: 14 April 2016.

Accepted for publication: 18 May 2016.

This work is licensed under a Creative Commons Attribution NonCommercial 4.0 License (CC BY-NC 4.0).

CCopyright D. Martolini et al., 2017

Licensee PAGEPress, Italy

Italian Journal of Medicine 2017; 11:37-40

doi:10.4081/itjm.2017.728 quired pneumonia (CAP). ${ }^{1}$ It is diagnosed in $5-12 \%$ of adults who present to General Practitioners with symptoms of lower respiratory tract infection, and 22$42 \%$ of these are admitted to hospital, where the mortality rate is between $5 \%$ and $14 \%$. Between $1.2 \%$ and $10 \%$ of adults admitted to hospital with CAP are managed in an intensive care unit, and for these patients the risk of dying is more than $30 \%$. More than half of pneumonia-related deaths occur in people older than 84 years. At any time $1.5 \%$ of hospital inpatients in England have a hospital-acquired respiratory infection, more than half of which are hospital-acquired pneumonia (HAP) not associated with intubation. HAP is estimated to increase hospital stay by about 8 days and has a reported mortality rate that ranges from 30 to $70 \%$. Variations in clinical management and outcome occur across the UK.

Illness scores such as pneumonia severity index (PSI) and CURB-65 (confusion, urea, respiratory rate, blood pressure, age $\geq 65$ ) are commonly used to estimate the severity and prognosis of patients affected by CAP. ${ }^{2}$ B-type natriuretic peptide (BNP) and its N-terminal fragment (NT-proBNP) are currently used to facilitate the diagnosis of heart failure (HF) and provide prognostic information in patients presenting with acute coronary syndromes. Cardiac troponins are biomarker for myocardial injury and together with BNP have been demonstrated to provide independent prognostic information in patients hospitalized with acute coronary syndromes. ${ }^{3}$ As cardiac troponin can be used to stratify risk in patients with pneumonia ${ }^{4,5}$ we hypothesized there is a good correlation between NT-pro-BNP as a reliable marker of myocardial stress and also of sepsis-induced myocardial depression and the pneumonia illness scores 
PSI and CURB-65. Moreover, we were also interested in evaluating any correlation with procalcitonin (PCT), well-known biomarker of sepsis due to bacterial sustained infection.

\section{Materials and Methods}

Twenty-three patients [age $79 \pm 15$ standard deviation (SD); M/F 8/15, CURB-65 2.2 \pm 0.9 SD, PSI $118 \pm 38$ SD, PCT $3.9 \pm 5$ ] with a diagnosis of pneumonia hospitalized in our Internal Medicine Unit were retrospectively evaluated. The diagnosis of pneumonia was made in the presence of pulmonary consolidation on chest X-ray or computed tomogaphy (CT) scan along with at least two of the following criteria: fever, cough with sputum or dyspnea, increased white blood cell count, and acute respiratory failure or worsening hypoxemia. NT-proBNP was measured in the first 72 $\mathrm{h}$ of hospitalization at the Emergency Department. Chest X-ray or CT-scan were also performed. CURB65 and PSI were calculated and correlation with the inflammatory biomarker investigated.

\section{Statistical analysis}

Microsoft Excel for Windows was used for general statistic. GraphPad Prism 2007 for Windows was utilized to evaluate the normal distribution of data. Nonparametric correlation (Spearman) was investigated among the variables of interest because they were not normally distributed. $\mathrm{P}<0.05$ was deemed significant. An $r$ between 0.3 and 0.7 and an $r>0.7$ show a moderate and a strong correlation respectively. Mean and $\mathrm{SD}$ were calculated for the variables of interest.

\section{Results}

NT-proBNP showed a moderate statistically significant correlation with both PSI and CURB-65 (NTproBNP vs PSI, Spearman correlation, $\mathrm{r}=0.42, \mathrm{P}<0.05$; Figure 1, NT-proBNP vs CURB-65, Spearman correlation, $r=0.46, \mathrm{P}<0.05$; Figure 2 ). A statistically significant moderate correlation, was observed also with PCT $(\mathrm{r}=0.46 ; \mathrm{P}<0.05$; Figure 3$)$. These correlations were confirmed also when patients with a diagnosis of HF ( $\mathrm{n}=11$ with $\mathrm{HF} ; \mathrm{n}=12$ without $\mathrm{HF}$ ) where excluded from the analysis, even if the correlation did not reach the statistical significance (Figures 4-6).

\section{Discussion}

Pneumonia is a widespread disease, particularly in the elderly population and is frequently characterized by the systemic inflammatory response syndrome (SIRS), which can evolve in septic shock. Brain natri-

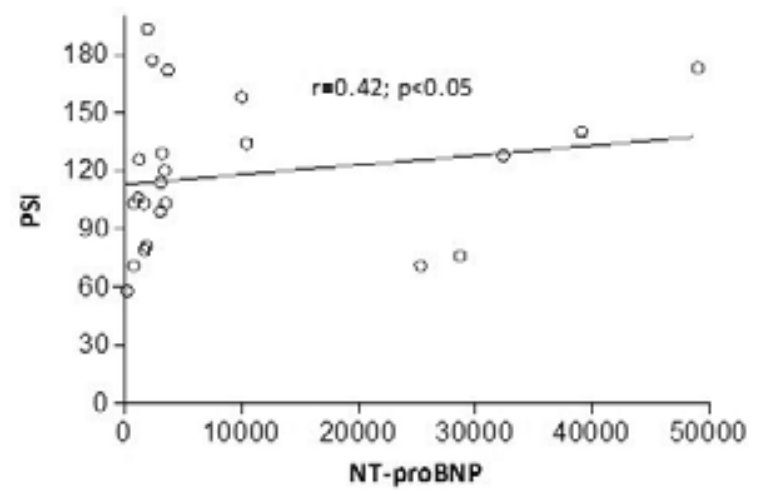

Figure 1. Correlation between pneumonia severity index (PSI) and the $\mathrm{N}$-terminal fragment of B-type natriuretic peptide (NT-proBNP) (Spearman, $n=23$ ).

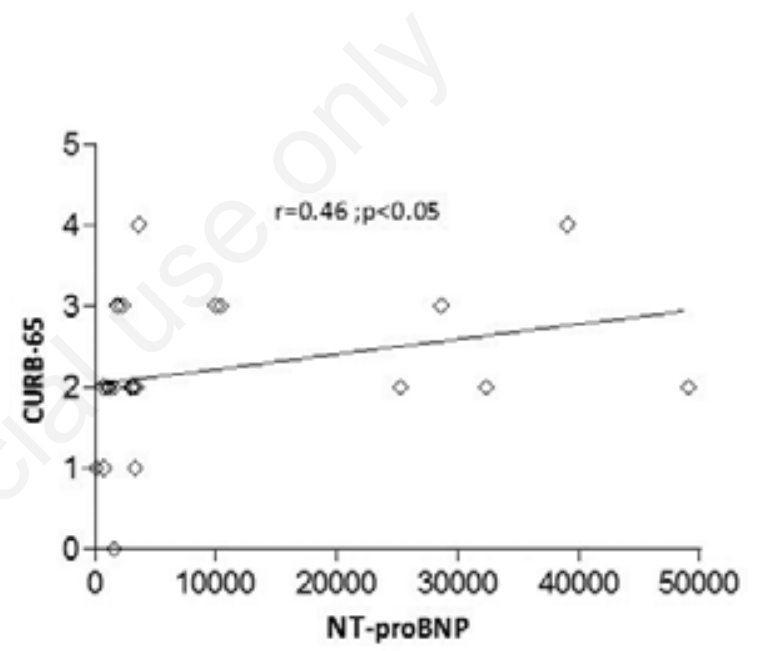

Figure 2. Correlation between CURB-65 (confusion, urea, respiratory rate, blood pressure, age $\geq 65$ ) and the N-terminal fragment of B-type natriuretic peptide (NT-proBNP) (Spearman, $\mathbf{n = 2 3 )}$.

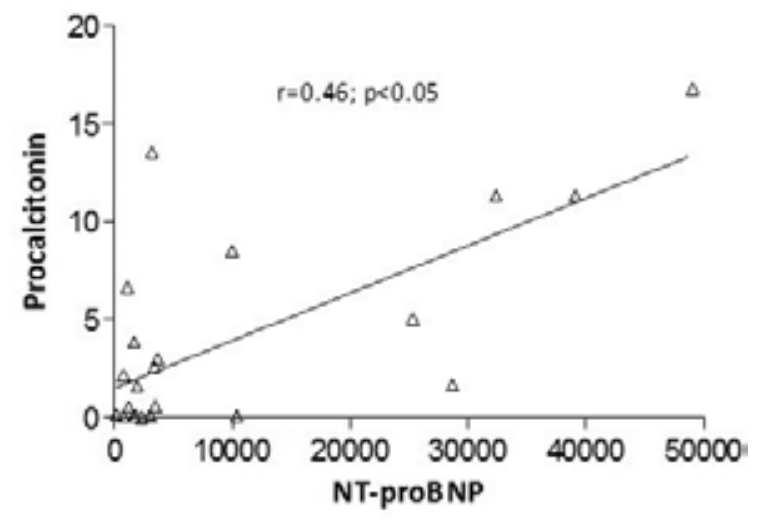

Figure 3. Correlation between procalcitonin and B-type natriuretic peptide and the $\mathrm{N}$-terminal fragment of $\mathrm{B}$-type natriuretic peptide (NT-proBNP) (Spearman, n=23). 


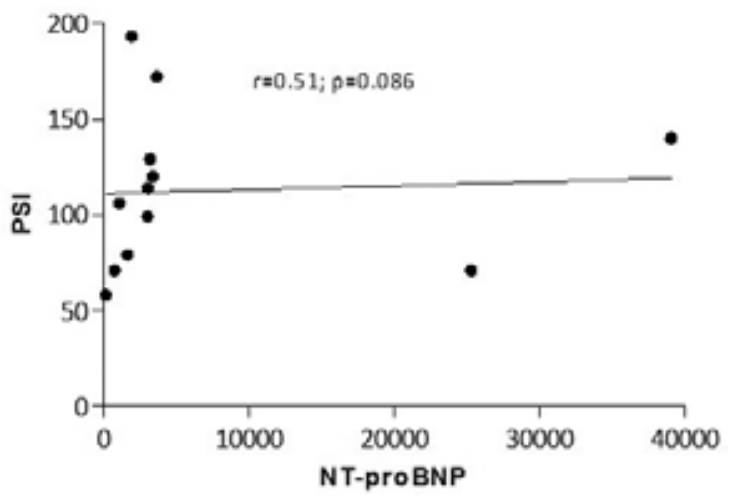

Figure 4. Correlation between pneumonia severity index (PSI) and the N-terminal fragment of B-type natriuretic peptide (NT-proBNP) (Spearman, $n=12$ ).

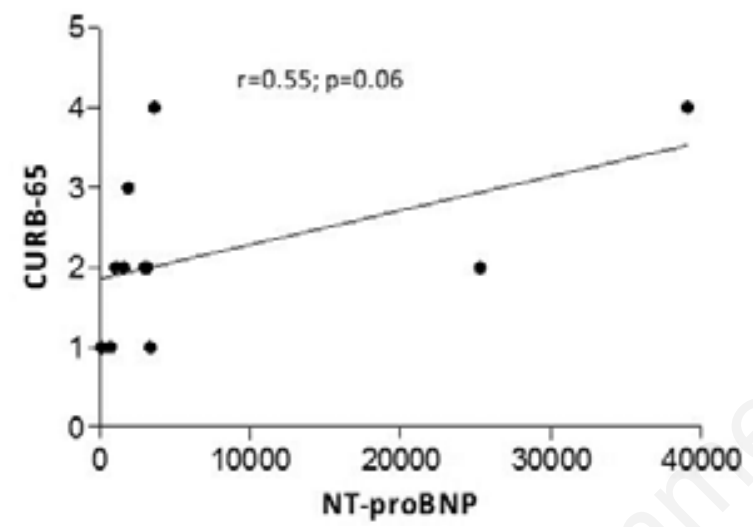

Figure 5. Correlation between CURB-65 (confusion, urea, respiratory rate, blood pressure, age $\geq 65$ ) and the $\mathrm{N}$-terminal fragment of B-type natriuretic peptide (NT-proBNP) (Spearman, $n=12)$.

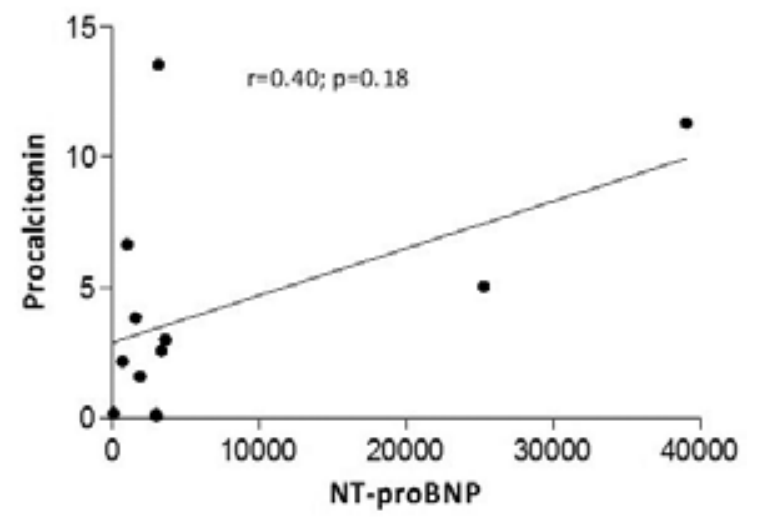

Figure 6. Correlation between procalcitonin and the N-terminal fragment of B-type natriuretic peptide (NT-proBNP) (Spearman, $n=12)$. uretic peptides along with other cardiac markers such as troponin have been found significantly elevated in sepsis and septic shock. ${ }^{6,7} \mathrm{Few}$ studies coming out in the last few years showed a good prognostic power of BNP to predict severity and prognosis of pneumonia. ${ }^{8-12} \mathrm{~Pa}-$ tients with pneumonia frequently face hypoxemia or respiratory failure which can be just part of the more complex SIRS and which can affect heart function. NTproBNP is synthesized in myocytes from the ventricles in relation to the severity of ventricular dysfunction and promotes the renal excretion of sodium (natriuresis) and water (diuresis), causes vasodilatation by relaxing vascular smooth muscle cells, improves diastolic relaxation (lusitropy) and decreases myocardial fibrosis. ${ }^{13}$ According to previous reports, our study shows that in patients with pneumonia hospitalized in an internal medicine ward there is a good and moderate correlation between NT-proBNP and the illness score PSI and CURB-6. These scores have demonstrated to be able to evaluate the severity and prognosis of patients with pneumonia and are suggested by the guidelines as tool to take into consideration, also to decide the setting where to treat the patients affected by pneumonia. ${ }^{2}$ In our study, we also showed that correlation between NT-proBNP and these scores is probably independent from a previous diagnosis of HF even if the statistical significance was not reached. A good correlation was also demonstrated with the biomarker PCT that is used as a diagnostic and severity marker in sepsis due to bacterial infection. Because NT-proBNP has been found elevated in sepsis the correlation with PCT supports our study hypothesis.

\section{Limitations}

Our study has several limitations. At first instance the number of patients is small, more female are represented, and the number of patients without a diagnosis of HF is even smaller. Secondly it is a retrospective study with all the limitations this implies. Thirdly we did not evaluate other factors, which could influence the level of NT-proBNP as age, anemia, hypoxemia or other comorbidities such as chronic obstructive pulmonary disease, acute coronary syndromes, renal failure, and others, and we did not evaluate the impact of a raised NT-proBNP on mortality. Fourthly there is not a group control, with the same characteristics and without pneumonia.

\section{Conclusions}

NT-proBNP showed a moderate and significant correlation with the illness score PSI and CURB-65 and can be considered a useful tool in the Emergency Department and Internal Medicine Ward to evaluate the severity and prognosis of patients with pneumonia and to decide the appropriate setting where to treat them. 


\section{References}

1. National Institute for Health and Care Excellence (NICE). Pneumonia in adults: diagnosis and management. Clinical guideline [CG191] - Published date: December 2014. Available from: https://www.nice.org.uk/guidance/cg191

2. Mandell LA, Wunderink RG, Anzueto A, et al. IDSA/ ATS Consensus guidelines on the management of community-acquired pneumonia in adults. CID 2007;44: S27-72.

3. Fonarow GC, Peacock WF, Horwich TB, et al. Usefulness of B-type natriuretic peptide and cardiac troponin levels to predict in-hospital mortality from ADHERE. Am J Cardiol 2008;101:231-7.

4. Moammar MQ, Ali MI, Mahmood NA, et al. Cardiac troponin I levels and alveolar-arterial oxygen gradient in patients with community-acquired pneumonia. Heart Lung Circ 2010;19:90-2.

5. Lee YJ, Lee H, Park JS, et al. Cardiac troponin I as a prognostic factor in critically ill pneumonia patients in the absence of acute coronary syndrome. J Crit Care 2015;30:390-4.

6. Post F, Weilemann LS, Messow CM, et al. B-type natriuretic peptide as a marker for sepsis-induced myocardial depression in intensive care patients. Crit Care Med 2008;36:3030-7.

7. Falcone M, Corrao S, Venditti M, et al. Performance of
PSI, CURB-65 and SCAP scores in predicting the outcome of patients with community-acquired and healthcare-associated pneumonia. Intern Emerg Med 2011;6:4 31-6.

8. Chang CL, Mills GD, Karalus NC, et al. Biomarkers of cardiac dysfunction and mortality from community-acquired pneumonia in adults. PLoS One 2013;8:e62612.

9. Nowak A, Breidthardt T, Dejung S, et al. Natriuretic peptides for early prediction of acute kidney injury in community-acquired pneumonia. Clin Chim Acta 2013;18;419:67-72.

10. Hodgson D, Nee P, Sultan L. Towards evidence-based emergency medicine: best BETs from the Manchester Royal Infirmary. BET 4: Prognostic value of B-type natriuretic peptides (BNP and NT-proBNP) in communityacquired pneumonia. Emerg Med J 2012;29:856-7.

11. Jeong KY, Kim K, Kim TY, et al. Prognostic value of $\mathrm{N}$-terminal pro-brain natriuretic peptide in hospitalised patients with community-acquired pneumonia. Emerg Med J 2011;28:122-7.

12. Leli C. Utility of brain natriuretic peptide as prognostic marker in community-acquired pneumonia and chronic obstructive pulmonary disease exacerbation patients presenting to the emergency department. Infez Med 2011;19:235-40.

13. Felker GM, Petersen JW, Mark DB. Natriuretic peptides in the diagnosis and management of heart failure. CMAJ 2006; 175:611-61. 